\title{
Originales
}

\section{Decisión del momento de extubación del postoperado de cirugía maxilofacial en la unidad de cuidados intensivos}

\author{
E. CURIEL BALSERA, M.A. PRIETO PALOMINO, J. MUÑOZ BONO, \\ M.D. ARIAS VERDÚ, J. MORA ORDÓÑEZY G. QUESADA GARCÍA
}

Unidad de Cuidados Intensivos. Hospital Regional Universitario Carlos Haya. Málaga. España.

Objetivo. Evaluar el momento de extubación de los pacientes postoperados de cirugía maxilofacial (CMF) que requieren ingreso en la unidad de cuidados intensivos ( $\mathrm{UCl}$ ) y relacionarlo con las complicaciones acaecidas durante su estancia.

Diseño. Estudio observacional prospectivo.

Ámbito. UCI de un hospital de tercer nivel.

Pacientes y métodos. Se estudió a todos los pacientes sometidos a CMF ingresados en UCI para manejo postoperatorio inmediato, desde febrero de 2007 hasta marzo de 2008. Se registraron variables clínicas y demográficas de los pacientes, variables anestésicas previas al acto quirúrgico y tiempos de ventilación mecánica y complicaciones inmediatas durante su estancia en UCI.

Resultados. Se registraron 102 pacientes durante el periodo del estudio. Se extubó precozmente (dentro de las primeras 4 h) a $58(55,8 \%)$ pacientes. La tasa general de complicaciones fue del $12,5 \%$. La duración de la ventilación mecánica fue mayor en la cirugía que requirió vaciamiento ganglionar cervical ( $p=0,003$ ). Encontramos relación $(p=0,03)$ entre la presencia de alguna complicación y la extubación tardía (odds ratio: 3,78 ; intervalo de confianza del 95\%, 1,16-12,31). El análisis multivariable reveló que son predictores de complicaciones la cirugía que incluye vaciamiento ganglionar y la extubación tardía.

Conclusiones. En nuestra serie, el destete tardío y la cirugía que conlleva vaciamiento ganglionar cervical fueron factores relacionados con morbilidad. A pesar de que la extubación del postoperado de CMF pueda parecer arriesgada en determinados casos y en las primeras horas,

Correspondencia: Dr. E. Curiel Balsera

Velez-Málaga, 6. 1. ${ }^{\circ}$ D. 29016 Málaga. España.

Correo electrónico: emiliouci@ya.com

Manuscrito aceptado el 17-11-2008. no disponemos de datos consistentes para mantener la ventilacion mecánica más allá de lo preciso para la recuperación anestésica.

PALABRAS CLAVE: Postoperatorio de cirugía maxilofacial. Vaciamiento ganglionar cervical. Complicaciones. Extubación precoz

\section{DECISION ON THE TIME FOR POST-OPERATIVE EXTUBATION OF MAXILLOFACIAL SURGERY PATIENT IN THE INTENSIVE CARE UNIT}

Objective. Evaluate moment of extubation in maxillo-facial post-operative patients admitted to an intensive care unit (ICU) and analyze early complications during their stay.

Design. An observational and prospective study.

Setting. Third level hospital ICU.

Patients and methods. All patients we underwent maxillo-facial surgery and admitted to the ICU for immediate post-operative care from February 2007 to March 2008 were studied. Demographic and clinical data variables of the patients, anesthesic variables prior to surgery and mechanical ventilation and postoperative complications during their stay in the ICU were recorded.

Results. A total of 102 patients were collected during the study. Of these, $58(55.8 \%)$ patients were extubated early (within the first 4 hours of admission). Global rate of complications was $12.5 \%$. Length of mechanical ventilation was longer in patients who required cervical lymph node extraction $(p=0.0031)$. We found an association between complications and late extubation $(p=0.034$; OR = 3.78; 95\% Cl, 1.16-12.31). The multivariant study showed that late extubation and surgery that required lymph node extraction are predictors of complications. 
Conclusions. In our series, late extubation and the need for cervical lymph node extraction were independent risk factors for complications in ICU. Although early extubation may be hazardous in some cases in the first hours, we have no consistent data to maintain mechanical ventilation longer than needed to recover from the anesthesia.

KEY WORDS: Fast-track. Maxillofacial surgery. Ventilator weaning. Postoperative care.

\section{INTRODUCCIÓN}

Se ha propuesto que la ventilación prolongada profiláctica podría reducir, en algunos postoperados, la incidencia de complicaciones respiratorias, aunque esta pauta de actuación es muy controvertida ${ }^{1}$. En la pasada década han aflorado más publicaciones sobre fast track o técnica de recuperación precoz, que no es más que una aproximación y planificación multimodal del periodo postoperatorio que tiene como objetivo no prolongar la intubación traqueal ni la ventilación mecánica. Las series más amplias versan sobre el postoperatorio de cirugía cardiovascular de pacientes adultos ${ }^{2}$, aunque hay artículos documentados sobre otro tipo de postoperatorios ${ }^{3,4}$, y también sobre pacientes pediátricos ${ }^{5,6}$.

Hay escasas referencias bibliográficas acerca del momento idóneo de extubación en el postoperado de cirugía maxilofacial (CMF) que ingresa en la unidad de cuidados intensivos (UCI), las cuales, además, datan de casi dos décadas ${ }^{7,8}$.

La extubación precoz del postoperado de CMF puede ser arriesgada en algunas ocasiones por la necesidad de reintubar a un paciente con cambios quirúrgicos en la orofaringe, con limitación de la apertura bucal o con sangrado o edema glótico, entre otras circunstancias adversas. El cerclaje elástico en vez del metálico, junto con el uso de anestésicos de menor vida media, ha contribuido a que la mayoría de estos pacientes sean extubados precozmente en la UCI y, a veces, en el mismo quirófano ${ }^{9}$. No hay un marcador específico en estos pacientes para cuantificar el grado de inflamación postoperatoria ni tampoco la posibilidad de prever posibles complicaciones por afección de la vía aérea superior. Sin embargo, como práctica habitual, utilizamos esteroides con fines antiinflamatorios y tratamos de extubar lo más precozmente a los pacientes.
Analizamos en nuestra serie el momento de la extubación del postoperado de CMF que ingresa en la UCI y su posible relación con la morbilidad del paciente.

\section{PACIENTES Y MÉTODOS}

Estudio observacional, prospectivo, de todos los pacientes ingresados en UCI tras ser intervenidos de CMF, desde febrero de 2007 hasta marzo de 2008. Se analizaron variables clínicas y demográficas de los pacientes, variables anestésicas previas al acto quirúrgico, como el grado de riesgo quirúrgico de la Sociedad Americana de Anestesiología (ASA) (tabla 1), o la previsión de una intubación dificultosa según las clasificaciones de Mallampati o Cormack (fig. 1). Además, se registró el tipo de intubación, la necesidad o no de fibrobroncoscopio y la duración del acto quirúrgico. Finalmente, se midieron variables en la UCI como la duración en horas de la ventilación me-

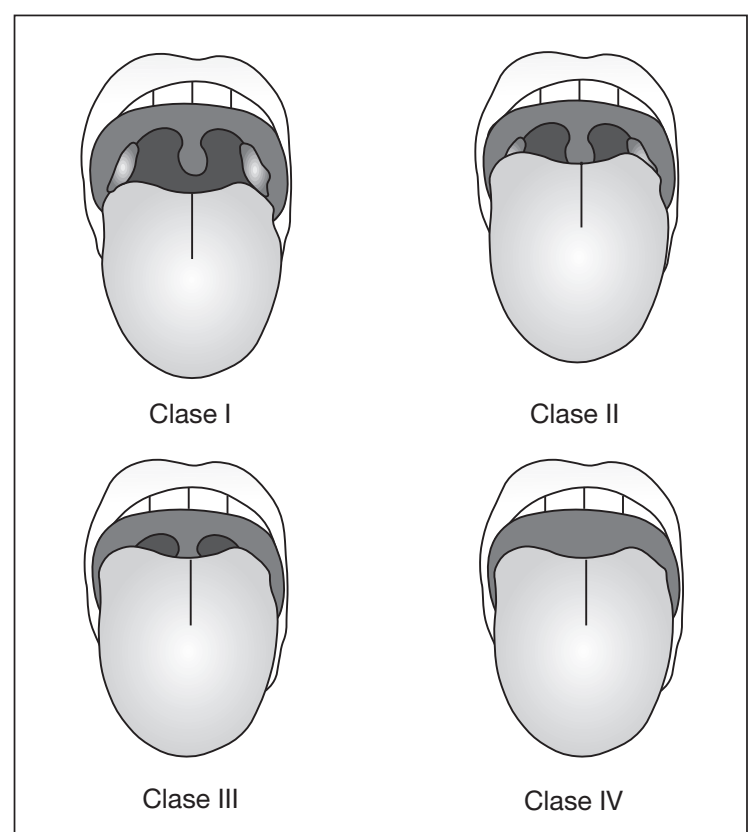

Figura 1. Clase I: visualización del paladar blando, la úvula y los pilares anterior y posterior de la faringe. Clase II: visualización del paladar blando y la úvula. Clase III: visualización del paladar blando y la base de la úvula. Clase IV: el paladar blando no está totalmente visible.

\section{TABLA 1. Clasificación de la American Society of Anesthesiologists (ASA)}

\begin{tabular}{|ll|}
\hline ASA I & $\begin{array}{l}\text { No se observan alteraciones orgánicas, fisiológicas ni bioquímicas. La enfermedad se limita a la cirugía que va a ser } \\
\text { realizada y ésta no implica ningún trastorno sistémico }\end{array}$ \\
ASA II & $\begin{array}{l}\text { Enfermedad sistémica leve a moderada, sin afección de órgano diana o limitación funcional } \\
\text { ASA III }\end{array}$ \\
Enfermedad sistémica severa con limitación funcional \\
ASA IV & Enfermedad sistémica severa con riesgo vital que no siempre es corregible por la cirugía \\
ASA V & Paciente moribundo con menos de 24 h de expectativa de vida sin cirugía \\
\hline
\end{tabular}


cánica, modos ventilatorios o las complicaciones precoces: respiratorias, cardíacas, hemáticas, neurológicas o renales.

Los diferentes tipos de cirugía se codificaron en 3 grupos, según si afectaba exclusivamente a regiones óseas (exéresis maxilares, mandibulares o del macizo facial), a partes blandas orofaríngeas o linguales (glosectomías, suelo de boca...) o si además la cirugía conllevaba un vaciamiento ganglionar cervical.

\section{DEFINICIONES OPERATIVAS DE VARIABLES}

- Complicación respiratoria: necesidad de reintubación o utilización de ventilación mecánica no invasiva tras una extubación.

- Complicación cardíaca: presentar un síndrome coronario agudo tras el acto quirúrgico o taquiarritmias o bradiarritmias significativas que requirieron tratamiento farmacológico o eléctrico.

- Complicación hemodinámica: presentar hipotensión mantenida que requiere más de 21 de fluidoterapia o hemoderivados o el empleo de fármacos vasoactivos.

- Complicación neurológica: aparición de un accidente cerebrovascular agudo (ACVA) en el postoperatorio inmediato.

- Complicación renal: aparición de fallo renal agudo de cualquier etiología, definido por la elevación al doble del valor de creatinina basal.

- Extubación precoz: la realizada dentro de las primeras $4 \mathrm{~h}$ desde el ingreso en UCI.

Los criterios de extubación empleados fueron: presentar adecuado nivel de conciencia con reflejo tusígeno presente, $\mathrm{FiO}_{2}<0,4$, presión positiva espiratoria final $(\mathrm{PEEP}) \leq 6 \mathrm{cmH}_{2} \mathrm{O}$, esfuerzo respiratorio espontáneo con frecuencia respiratoria no mayor de 30 por min, presión inspiratoria máxima menor de -20 $\mathrm{cmH}_{2} \mathrm{O}$, estabilidad hemodinámica y ausencia de acidosis respiratoria en gasometría. En los casos en que se consideró necesario se realizó también el test de fuga aérea para valorar la posibilidad de obstrucción de la vía aérea superior.

Tanto el registro informatizado de las variables como el análisis estadístico de los datos se realizaron con el programa estadístico SPSS (Statistical Package for Social Sciences), versión 15. Las variables cualitativas se expresaron como porcentajes y frecuencia absoluta. Las variables cuantitativas se expresaron como media y desviación estándar, o mediana e intervalo intercuartílico para variables asimétricas. Para los contrastes de hipótesis se han utilizado las pruebas de la $\chi^{2}$ o la t de Student, según el caso. Por último, se ha realizado un análisis multivariable mediante regresión logística binaria para identificar factores predictores. La significación estadística se estableció considerando un error alfa $<5 \%$.

Se informó a los participantes y/o familiares de la naturaleza de la investigación, y se les garantizó absoluta confidencialidad de los datos obtenidos, lo cual se puso en conocimiento de la Comisión de Ética del hospital.

\section{RESULTADOS}

Se registraron 102 pacientes, cuyas variables clínicas y demográficas se muestran en la tabla 2. Se extubó precozmente a $58(55,8 \%)$ pacientes, es decir, dentro de las primeras $4 \mathrm{~h}$ desde su ingreso en UCI. La tasa general de complicaciones fue del 12,5\% (13 casos); la mayor parte de ellas fueron de origen respiatorio, principalmente por obstrucción de la vía aérea superior (por edema o hemorragia) e hipoventilación por persistencia de efectos sedoanalgésicos residuales. Dos pacientes presentaron sangrado excesivo que precisó reanimación enérgica y politransfusión. Un paciente presentó fibrilación auricular rápida y otros 4 sufrieron algunas de complicaciones concomitantes. Solamente se registró un fallecimiento, debido a una complicación séptica en un paciente de edad avanzada e inmunodeficiente.

TABLA 2. Variables clínicas y demográficas de los pacientes incluidos en el estudio

\begin{tabular}{|c|c|}
\hline Varones & $62(59,6 \%)$ \\
\hline Mujeres & $42(40,4 \%)$ \\
\hline Edad (años) & $52,61 \pm 18,9$ \\
\hline Cirugía programada/urgente & $98(94,2 \%) / 6(5,8 \%)$ \\
\hline Ventilación mecánica (h) & $4[3-14,75]$ \\
\hline ASA I & $20(19,6 \%)$ \\
\hline ASA II & 46) $45,1 \%$ ) \\
\hline ASA III & $36(35,3 \%)$ \\
\hline ASA IV y V & 0 \\
\hline Previsión de vía aérea difícil & $24(26,1 \%)$ \\
\hline Intubación con broncoscopio & $32(37,6 \%)$ \\
\hline \multicolumn{2}{|l|}{ Tipo intubación } \\
\hline Nasotraqueal & $73 \%$ \\
\hline Orotraqueal & $20 \%$ \\
\hline Horas de quirófano & $4,41 \pm 1,5$ \\
\hline \multicolumn{2}{|l|}{ Tipo de cirugía } \\
\hline $\begin{array}{l}\text { Paladar blando, faringe, lengua o } \\
\text { suelo de boca }\end{array}$ & $35(33,7 \%)$ \\
\hline $\begin{array}{l}\text { Paladar duro (maxilar), mandíbula } \\
\text { o huesos faciales }\end{array}$ & $39(37,5 \%)$ \\
\hline $\begin{array}{l}\text { Cirugía con vaciamiento } \\
\text { ganglionar cervical }\end{array}$ & $30(28,8 \%)$ \\
\hline Complicaciones & $13(12,5 \%)$ \\
\hline Respiratorias & 6 \\
\hline Hemodinámicas & 2 \\
\hline Cardíacas & 1 \\
\hline Varias & 4 \\
\hline Reintervención & $4(3,8 \%)$ \\
\hline Muerte & $1(1 \%)$ \\
\hline
\end{tabular}

y mediana [intervalo intercuartílico]. 
En general, la mediana de estancia fue $24 \mathrm{~h}$; el promedio de la estancia entre los extubados precozmente frente a los demás pacientes no presentó diferencias significativas $(\mathrm{p}=0,87)$. En cuanto a la duración de la ventilación mecánica, ésta fue signicativamente mayor en la cirugía oncológica que requirió vaciamiento ganglionar cervical unilateral o bilateral que en el resto de los casos $(\mathrm{p}=$ 0,031) (fig. 2). Asimismo, el promedio de duración en horas de cirugía fue significativamente mayor cuando se realizó un vaciamiento ganglionar cervical que en el caso de cirugía sobre el paladar blando

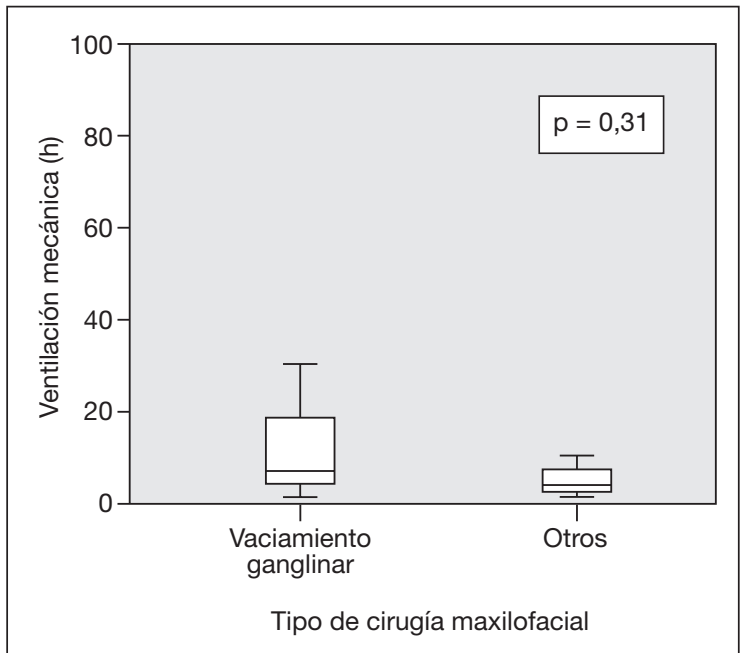

Figura 2. Tiempo de ventilación mecánica en el grupo de cirugía que incluyó vaciamiento ganglionar cervical y en las demás intervenciones quirúrgicas.

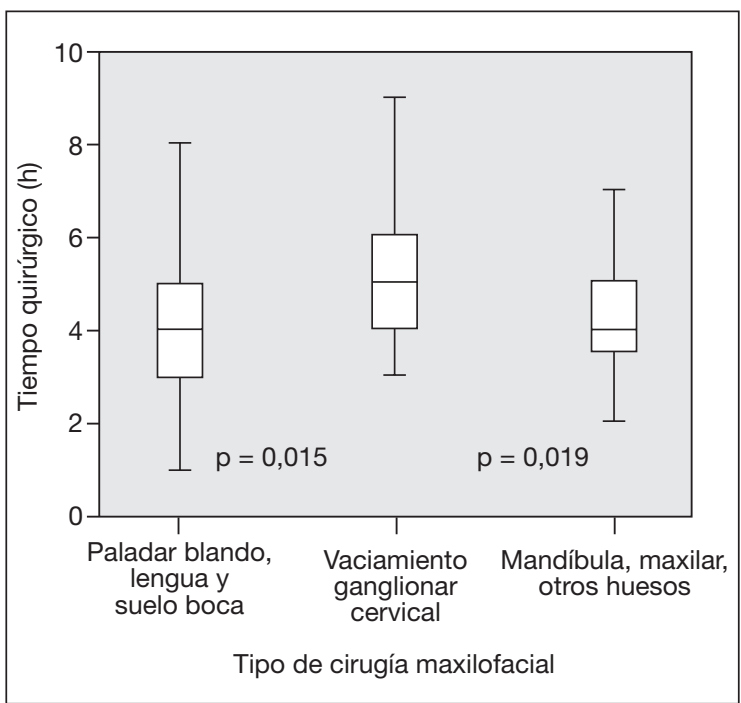

Figura 3. Duración del acto quirúrgico en los tres grupos de estudio establecidos. y la lengua $(\mathrm{p}=0,015)$ o sobre la mandíbula y el maxilar ( $\mathrm{p}=0.019)$ (fig. 3).

A pesar de que el tiempo quirúrgico y el de ventilación mecánica fueron mayores en el grupo de pacientes sometidos a vaciamiento ganglionar cervical, encontramos diferencias en el estado previo de salud, valorado mediante la clasificación de riesgo quirúrgico ASA, al encontrar menor escala ASA en este grupo de pacientes que en el que no se realizó vaciamiento ganglionar cervical $(\mathrm{p}=0,011)$.

Hubo relación significativa $(\mathrm{p}=0,034)$ entre la presencia de alguna complicación y la extubación tardía (odds ratio $[\mathrm{OR}]=3,78$; intervalo de confianza [IC] del 95\%, 1,16-12,31). El análisis multivariable reveló que son predictores de complicaciones la cirugía que incluye vaciamiento ganglionar y la extubación tardía (tabla 3 ).

\section{DISCUSIÓN}

La extubación traqueal no está exenta de complicaciones de diversa etiología, como las respiratorias (laringospasmo, obstrucción de la vía aérea, desaturación por hipoventilación, etc.). La extubación precoz del postoperado de CMF puede ser arriesgada, principalmente por el riesgo aumentado de fallo por obstrucción de la vía aérea y reincubación difícil. Se debe sopesar el riesgo/beneficio de extubar tempranamente o de mantener con sedoanalgesia y ventilación mecánica durante más tiempo.

En nuestra serie, los pacientes en que se llevó a cabo la extubación precoz presentaron significativamente menos complicaciones que aquellos a quienes se extubó más tardiamente, después de 5 h desde el ingreso en la UCI. Por tanto, el destete tardío fue un factor de riesgo de morbilidad, tanto en el estudio univariable como en el multivariable.

En la literatura no hemos encontrado ningún artículo reciente que haga referencia al momento adecuado de extubación del postoperado de cirugía maxilofacial. El publicado hace 20 años por Haber-Cohen et $\mathrm{al}^{8}$ también registró mayor número de complicaciones respiratorias en los extubados tardíamente (en este caso, más de $8 \mathrm{~h}$ ), si bien sólo incluyó a pacientes tras cirugía ortognática y, evidentemente, son periodos poco comparables en lo que se refiere al desarrollo tecnológico en monitorización, uso de respiradores y modos ventilatorios, entre otros aspectos.

La extubación precoz es un elemento importante del fast-track, y ha mostrado beneficios en las últimas dos décadas en cuanto a morbilidad o estancia hospitalaria en pacientes sometidos a resecciones pulmonare ${ }^{10}$, en la cirugía de aorta abdominal ${ }^{11}$, prostatectomía radical $^{12}$, carcinoma colorrectal ${ }^{13}$ o cirugía traumatológica de cadera y rodilla ${ }^{14}$, entre otras intervenciones quirúrgicas.

En nuestra serie, la decisión de extubar de forma precoz se tomó sobre la base de datos objetivos, como parámetros hemodinámicos, respiratorios y gasométricos, así como otros datos perioperatorios, como la dificultad de intubación del paciente transmitida por el anestesista, la necesidad o no de ayuda del fibro- 
TABLA 3. Factores predictores de complicaciones según el estudio multivariable

\begin{tabular}{|l|l|l|l|}
\hline & Beta & $\mathrm{p}$ & OR (IC del 95\%) \\
\hline Vaciamiento ganglionar & 1,302 & 0,012 & $3,67(1,1-12,11)$ \\
Extubación tardía & 1,214 & 0,02 & $3,36(1,01-11,2)$ \\
\hline
\end{tabular}

Beta: coeficiente de regresión; IC: intervalo de confianza; OR: odds ratio.

broncoscopio para la intubación, el uso obligado de la vía nasotraqueal por cuestiones de técnica quirúrgica, la necesidad de cerclajes o fijaciones maxilares que dificulten la apertura bucal y la duración y el tipo de cirugía. En estos casos de difícil decisión se utilizó el test de fuga aérea ${ }^{15} \mathrm{y}$ el fibrobroncoscopio para valorar la presencia de edema en tejidos blandos supraglóticos.

La extubación durante un plano anestésico intermedio, entre dormido y despierto, puede minimizar algunos efectos indeseables como la hipertensión arterial y la taquicardia, pero conlleva mayores complicaciones respiratorias, como hiperreactividad refleja de la región glótica y supraglótica, que incrementan el riesgo de laringospasmo ${ }^{16}$.

La cirugía que conllevó un vaciamiento cervical ganglionar supuso un incremento significativo del tiempo quirúrgico y también fue una variable independiente de la presencia de complicaciones, especialmente respiratorias. Por ello, en este tipo de intervenciónquirúrgicadeberáextremarselamonitorización y los cuidados postoperatorios. Asimismo, la extubación tardía en estos pacientes puede verse facilitada si se mantiene un tratamiento esteroideo agresivo que reduzca el grado de inflamación y edema tisular y tras comprobar que no haya hemorragia.

Por otra parte, si bien la mayoría de nuestros pacientes fueron extubados precozmente, esta circunstancia no tuvo implicación alguna en la mortalidad ni en la duración de la estancia en la unidad, con relación a los que se extubó de forma no precoz.

Dada la antigüedad de los trabajos publicados sobre el destete en el postoperatorio de cirugía maxilofacial y el número de casos incluidos en ellos, sería necesario plantear nuevos estudios prospectivos para adecuar nuestra práctica clínica a la mejor evidencia científica.

\section{BIBLIOGRAFÍA}

1. Shackford SR, Virgilio RW, Peters RM. Early extubation versus prophylactic ventilation in the high risk patient: a comparison of postoperative management in the prevention of respiratory complications. Anesth Analg. 1981;60:76-80.
2. Wong D, Cheng D, Kustra R, Tibshirani R, Karski J, CarrollMunro J, et al. Risk factors for delayed extubation, prolonged length of stay in the intensive care unit, and mortality in patients undergoing coronary artery bypass graft with fast-track cardiac anesthesia: A new cardiac risk score. Anesthesiology. 1999;91:936-44.

3. Berberat PO, Ingold H, Gulbinas A, Kleeff J, Muller MW, Gutt C, et al. Fast track -different implications in pancreatic surgery. J Gastrointest Surg. 2007;11:880-7.

4. Konstantinov IE, McNeil K, Yeung S, Fawcett J, Mullany D, Dunning J. Fast track recovery following en bloc heart-lung-liver transplantation in a patient with cystic fibrosis complicated by severe portal hypertension. Heart Lung Circ. 2008;17:154-6.

5. Marianeschi SM, Seddio F, McElhinney DB, Colagrande L, Abella RF, De la Torre T, et al. Fast-track congenital heart operations: a less invasive technique and early extubation. Ann Thorac Surg. 2000;69:872-6.

6. Vricella LA, Dearani JA, Gundry SR, Razzouk AJ, Brauer SD, Bailey LL. Ultra fast track in elective congenital cardiac surgery. Ann Thorac Surg. 2000;69:865-71.

7. Zulian MA, Chisum JW, Mosby EL, Hiatt WR. Extubation criteria for oral and maxillofacial surgery patients. J Oral Maxillofac Surg. 1989;47:616-20.

8. Haber-Cohen A, Rothman M. A survey of extubation practices following orthognathic surgery. J Oral Maxillofac Surg. 1988;46:269-71

9. Buckley MJ, Tulloch JF, White RP Jr, Tucker MR. Complications of orthognathic surgery: a comparison between wire fixation and rigid internal fixation. Int $\mathrm{J}$ Adult Orthodon Orthognath Surg. 1989;4:69-74.

10. Muehling BM, Halter GL, Schelzig H, Meierhenrich R, Steffen P, Sunder-Plassmann L, et al. Reduction of postoperative pulmonary complications after lung surgery using a fast track clinical pathway. Eur J Cardiothorac Surg. 2008;393:281-7.

11. Brustia P, Renghi A, Fassiola A, Gramaglia L, Della CF, Cassatella R, et al. Fast-track approach in abdominal aortic surgery: left subcostal incision with blended anesthesia. Interact Cardiovasc Thorac Surg. 2007;6:60-4.

12. Gralla O, Buchser M, Haas F, Anders E, Kramer J, Lein $\mathrm{M}$, et al. Fast-track laparoscopic radical prostatectomy. Urologe. 2008;2 [en prensa].

13. Kuthan D, Ludvik P, Podebradsky J, Korinek P. Our initial experience with the fast-track method in the colorectal carcinoma management. Rozhl Chir. 2008;87:125-7.

14. Husted H, Holm G, Jacobsen S. Predictors of length of stay and patient satisfaction after hip and knee replacement surgery: fast-track experience in 712 patients. Acta Orthop. 2008; 79:168-73.

15. De Bast Y, De Backer D, Moraine JJ, Lemaire M, Vanderborght C, Vincent JL. The cuff leak test to predict failure of tracheal extubation for laryngeal edema. Intensive Care Med. 2002;28:1267-72.

16. Donlon JV. Anestesia y cirugía oftamológica y otorrinolaringología. En: Miller R, editor. Anestesia. 2.a ed. Barcelona: Doyma; 1993. p. 640-4. 